\title{
Dynamic evolution of interplanetary shock waves driven by CMEs
}

\author{
P. Corona-Romero ${ }^{1,2}$ and J. A. Gonzalez-Esparza ${ }^{2}$ \\ ${ }^{1}$ Posgrado en Ciencias de la Tierra, Universidad Nacional Autonoma de Mexico, \\ Av. Universidad 2000, Mexico City, Mexico. \\ email: piter.cr@gmail.com \\ ${ }^{2}$ Insituto de Geofisica Michoacan, Universidad Nacional Autonoma de Mexico \\ Tzintzuntzan 310, Morelia, Mexico. \\ email: americo@geofisica.unam.mx
}

\begin{abstract}
We present a study about the propagation of interplanetary shock waves driven by super magnetosonic coronal mass ejections (CMEs). The discussion focuses on a model which describes the dynamic relationship between the CME and its driven shock and the way to approximate the trajectory of shocks based on those relationships, from near the Sun to 1 AU. We apply the model to the analysis of a case study in which our calculations show quantitative and qualitative agreements with different kinds of data. We discuss the importance of solar wind and CME initial conditions on the shock wave evolution.
\end{abstract}

Keywords. Sun: coronal mass ejections (CMEs), Shock waves, Sun: activity, Methods: analytical.

\section{Introduction}

A shock wave is an energetic compressive perturbation which propagates faster than the characteristic speed of the medium. An interplanetary shock wave transfers energy to the solar wind increasing its entropy and kinetic energy. From a macroscopic perspective, a shock wave can be regarded as a discontinuity which separates two distinct fluids whose properties are related by the so called jump conditions (Landau \& Lifshitz, 2005). Interplanetary shocks associated with CMEs are an important issue due to their impact on the Earth magnetic conditions and increase our contingency plans.

In Heliophysics there are two main conditions in which a shock wave evolves: driven and decaying. During the first one, the shock energy transferred to the medium is restored by a driver. On the other hand, during the decaying, the shock constantly loses energy by transferring it to the medium. Corona-Romero \& Gonzalez-Esparza (2011) studied the dynamical evolution of shocks associated with CMEs using numerical simulations and analytical models. They found that the CME-shock evolution presents three different dynamic phases: driving, decoupling, and decaying. These dynamic phases are defined by the evolution of the linear momentum between the CME and the shock wave, i.e. the linear momentum flux through the plasma sheath (ambient wind modified by the shock propagation).

Figure 1 shows a sketch of the CME-shock system at the driving (a) and decaying (b) phases. During the driving phase the CME transfers momentum to the sheath, which results in a strong compression of the plasma sheath (darker gray region in panel a). On the other hand, during the decaying phase the CME is no longer transferring momentum to the sheath. Thus, during the decaying phase, the compression on the sheath decreases (panel b) and the distance between the CME and the shock increases. The decoupling 
process bounds these two opposite behaviors, during which the dynamic disconnection between driver (CME) and shock wave occurs by sheath relaxation.

\section{CME-shock propagation}

We can approximate the trajectory of shocks and CMEs on the basis of the three phases introduced above. In Figure 2 we present a comparison between the data and the calculations of the speeds (left panel) and heliocentric positions (right panel) for the May 132005 CME-shock event. The speed-position temporal evolution was studied previously by Bisi et al. (2010, and references there in) by combining different measurements such as white light coronagraph images (diamonds), the frequency drift of kilometric type II spectrum (triangles), interplanetary scintillation (squares and asterisks), and in situ spacecraft observations (crosses). Though the data do not refer to the same structure, they are related to the same phenomena (CME-shock event) and give us an approximation of the CME and shock propagations.

At the earlier moments of the CME and shock propagation, during the driving phase, the CME-shock configuration can be approximated by a quasi-spherical figure which drives a bow shock (Ontiveros \& Vourlidas, 2009). In this phase we approximate the CME-shock configuration by quasi-stationary bow shock. In this case we observe two characteristics: (1) the standoff distance (shortest distance between the CME and the shock, see Figure 1a) is a function of the magneto-sonic Mach number and the CME geometry, and (2) the standoff distance is almost constant, i.e. the CME and the shock share the same speed. These characteristics can be appreciated in Figure 2a, where CME and shock speeds appear similar (between the solid and dashed vertical gray lines) and, thus, the distance between them is constant. The duration of this period highly depends on the CME initial kinetic energy and on the solar wind conditions between them (Corona-Romero \& Gonzalez-Esparza, 2011).

It is well accepted that fast CMEs arrive at $1 \mathrm{AU}$ with slower speeds than their initial coronagraph counterparts (Gopalswamy, 2000), i.e. in general fast CMEs decelerate in the interplanetary medium. Once a fast CME decelerates, it is not able to transfer momentum
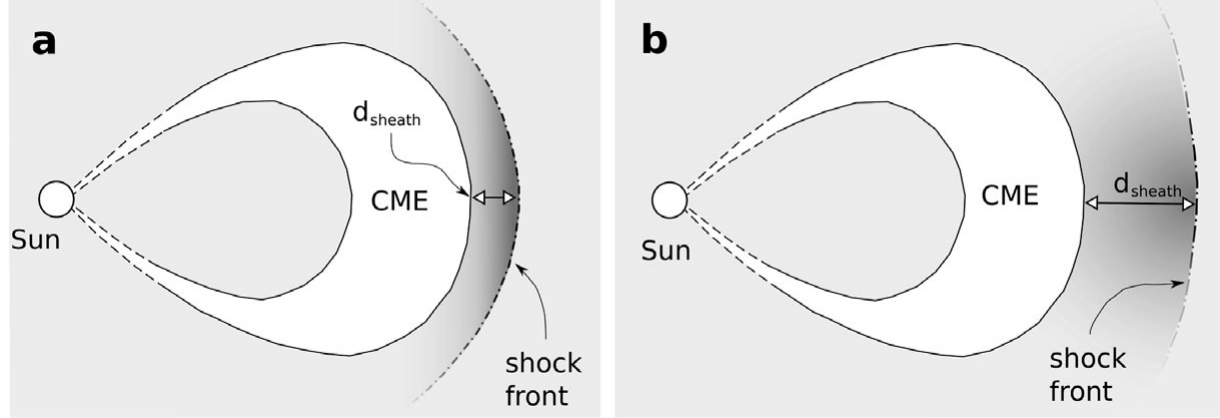

Figure 1. Sketch of a fast CME-shock system propagating through the interplanetary medium showing the driving (a) and decaying (b) configurations. From left to right, the Sun (white circle), the solar wind (light gray), the CME (white croissant-like shape), the plasma sheath or shocked solar wind (darker gray) and the shock front (dashed-dotted black line), respectively. The gray color shades represent the density of the ambient solar wind, being the darker regions (plasma sheath) the denser ones. (a) The CME drives the shock front (the dashed-dotted black line is clearly defined), the sheath is highly compressed and the standoff distance $\left(d_{\text {sheath }}\right)$ is constant. (b) The shock is getting dissipated (the dashed-dotted black line is vanishing), the compression on the sheath material decreases and the standoff distance grows. 
to the sheath, circumstance which triggers the decoupling process. In this process, with the absence of the CME momentum flux, the plasma sheath begins to relax; such a relaxation propagates throughout the sheath reaching the shock front. In Figure 2 (left panel) we see that along the decoupling process (limited by the dashed and dotted-dashed vertical gray lines) the shock preserves its driving speed and together with the CME deceleration produces an increasing in the plasma sheath's volume (with the standoff distance growing). The duration of this phase is proportional to the quotient of the driving standoff distance and the magneto-sonic speed inside the plasma sheath.

When the relaxation has propagated all over the plasma sheath width (the standoff distance) the shock speed begins to decrease, denoting the start of the decaying phase. During the latter phase, the CME is still decelerating, tending to reach the ambient solar wind speed and the shock decays into a magneto-sonic perturbation. In Figure 2, (a) and (b) panels, we can identify two characteristics in the decaying phase: (1) the standoff distance keeps increasing with time (the shock is faster than the CME) and (2) both velocities are lower than those of their driving counterparts.

\section{Comparison with observations}

According with our dynamic model, during the driving phase and the decoupling process the shock front propagates with the CME leading edge speed. On the other hand, during the decaying phase the shock heliocentric position $\left(r_{\text {shock }}\right)$ and speed $\left(v_{\text {shock }}\right)$ decay as $r_{\text {shock }} \propto t^{2 / 3}$ and $v_{\text {shock }} \propto r_{\text {shock }}^{-1 / 2}$, respectively, with $t$ the time (Cavaliere \& Messina, 1976). The previous expressions have been proposed by Smart \& Shea (1985)
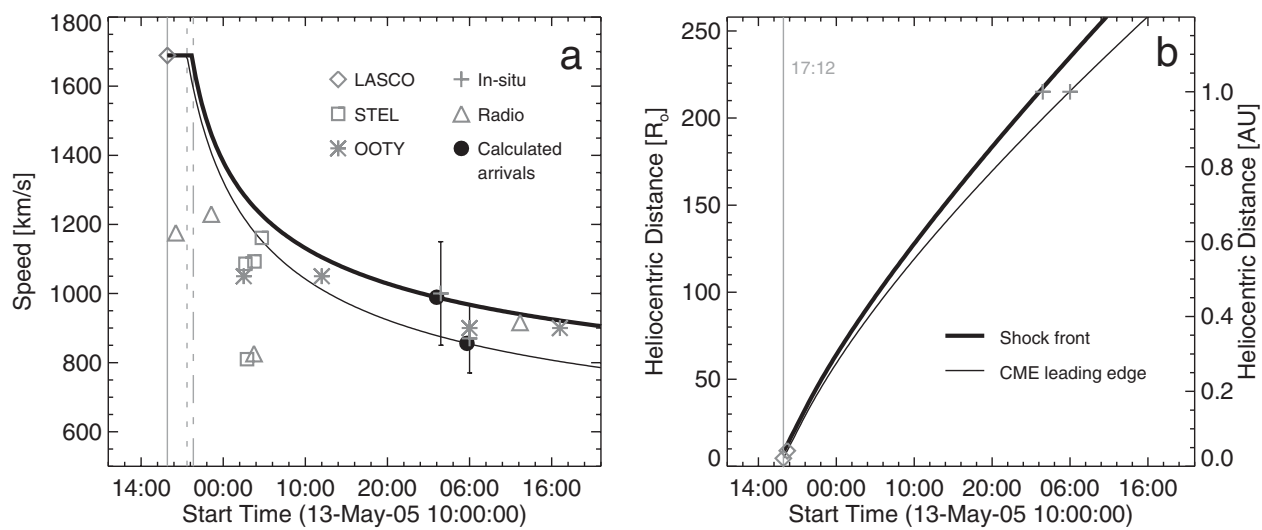

Figure 2. Comparison between the results of the analysis and the data for May 132005 event. (a) Calculated CME and shock speeds (black lines) vs. time. (b) Calculated CME and shock heliocentric distances vs. time (black lines). The different symbols in panels (a) and (b) indicate data from coronagraph (LASCO) images, speeds inferred from radio emission, in situ and interplanetary scintillation (STEL and OOTY) measurements. The driving phase lasts 2.38 hours (dashed vertical gray line, panel a) and the decoupling process 0.78 hours (dotted-dashed vertical gray line, panel a). To calculate the CME's nose trajectory we follow the description by Corona-Romero \& Gonzalez-Esparza (2011) with $\Delta t=0.50 h, a=5.01, c=8, r_{0}=4.57 R_{o}$ and $v_{0 \mathrm{cme}}=1689 \mathrm{~km} / \mathrm{s}$. The ambient wind conditions at $1 \mathrm{AU}$ were $n_{p}=4 \mathrm{cc}, T_{p}=130 \times 10^{3} \mathrm{~K}$, $|B|=6 \mathrm{nT}$, and $v_{s w}=420 \mathrm{~km} / \mathrm{s}$, respectively. The shock front propagation was calculated according the respective solution by Cavaliere \& Messina (1976). The driving standoff distance and the CME radius were calculated using the models of Farris \& Russell (1994) and Bothmer \& Schwenn (1998), respectively. 
and corroborated by Pintér \& Dryer (1990) in order to calculate transit times and to describe the propagation of interplanetary shocks associated with solar flares.

In this case study we find reasonable agreement between the data and the calculated positions and speeds. In Figure 2 we see that in situ (gray crosses) arrival speeds (panel a) and travel times (panel b) are quantitatively similar to their analytical counterparts (black circles). On the other hand, the interplanetary scintillation (OOTY and STEL) and the radio burst data show qualitative agreements with the predicted trajectory of the sheath (limited by the solid and dashed black lines).

The shock wave is not driven by the CME when it arrives to the Earth's orbit. According to the data, the CME speed at the solar corona $(\approx 1690 \mathrm{~km} / \mathrm{s})$ is considerably slower than its $1 \mathrm{AU}$ counterpart $(\approx 800 \mathrm{~km} / \mathrm{s})$. On the other hand, the shock front speed at 1 $\mathrm{AU}(\approx 1100 \mathrm{~km} / \mathrm{s})$ is faster than the CME, but it is still slower than the initial speed of the CME. Both conditions are consistent with the signatures for the decaying phase.

Transit times (panel b) and arrival speeds (panel a) matched with in situ data and speed profiles were consistent with the different sets of data (panel b). According to our results, the driving phase and decoupling process last 3.2 hours approximately, while the CME-shock transit time to $1 \mathrm{AU}$ is larger than 30 hours. Thus, the CME-shock evolution, in this case study, spends most of its transit time in the decaying stage.

\section{Summary}

We described the dynamical phases (driving, decoupling and decaying) that might be present in the fast CME-interplanetary shock propagation. We also analyzed a case study by applying the dynamical phase descriptions. Our results showed quantitative and qualitative agreements with different kinds of data. The three-phases dynamical evolution described above may help to approximate the trajectory, transit times, and arrival speeds of interplanetary shocks associated with fast CMEs. In order to do so we require the initial conditions of the $\mathrm{CME}$ and solar wind, which are determinant on the shock evolution.

\section{Acknowledgments}

Pedro Corona Romero acknowledges the grant from CONACyT and the IAU support. J. A. Gonzalez Esparza thanks the support of the CONACyT 48494 and PAPIIT IN105310-3 projects.

\section{References}

Bisi, M. M., Breen, A. R., Jackson, B. V., Fallows, R. A., Walsh, A. P., Mikić, Z., Riley, P., Owen, C. J., Gonzalez-Esparza, A., Aguilar-Rodriguez, E., Morgan, H., Jensen, E. A., Wood, A. G., Owens, M. J., Tokumaru, M., Manoharan, P. K., Chashei, I. V., Giunta, A. S., Linker, J. A., Shishov, V. I., Tyul'Bashev, S. A., Agalya, G., Glubokova, S. K., Hamilton, M. S., Fujiki, K., Hick, P. P., Clover, J. M., \& Pintér, B. 2010, Solar Phys., 265, 49

Bothmer, V. \& Schwenn, R. 1998, Ann. Geophysicae, 16, 1

Cavaliere, A. \& Messina, A. 1976, ApJ, 209, 424

Corona-Romero, P. \& Gonzalez-Esparza, J. A. 2011, JGR, 116, A05104

Farris, M. H. \& Russell, C. T. 1994, JGR, 99, 17681

Gopalswamy, N., Lara, A., Lepping, R. P., Kaiser, M. L., \& Berdichevsky, D., St. Cyr, O. C. 2000, GRL, 27, 145

Landau, D. L. \& Lifshitz, M. E. 2005, Fluid Mechanics, Course of Theoretical Physics Vol. 6 (Edit. Elsevier). 
Ontiveros, V. \& Vourlidas, A. 2009, ApJ, 693, 267

Pintér, S. \& Dryer, M. 1990, Bull. Astron. Inst. Czechosl., 41, 137

Smart, D. F. \& Shea, M. A. 1985, JGR, 90, 183

\section{Discussion}

DANIEL GómEz: In MHD there are three types of shocks. What happens if the CME decelerates?

Pedro Corona-Romero: In general fast CMEs are associated with fast MHD shocks. When a fast CME begins to decelerate, its role as shock driver ends. Thus, the shock begins to decay and its speed decreases. The important point is to understand how a shock dissipates: Does it change from a fast to a slow shock and finally to an MHD wave? Does a fast shock directly dissipate into an MHD wave? Does turbulence play an important role? But those are more likely statistical physics topics than MHD ones. 